\title{
ECDC publishes its first data on antimicrobial consumption in Europe
}

K Weist (klaus.weist@ecdc.europa.eu) ${ }^{1}$, on behalf of the ESAC-Net participants and the Antimicrobial Resistance and Healthcare-associated Infections (ARHAI) programme at ECDC ${ }^{1}$

1. European Centre for Disease Prevention and Control (ECDC), Stockholm, Sweden

Citation style for this article:

Weist K, on behalf of the ESAC-Net participants and the Antimicrobial Resistance and Healthcare-associated Infections (ARHAI) programme at ECDC. ECDC publishes its first data on antimicrobial consumption in Europe. Euro Surveill. 2013;18(10):pii=20419. Available online: http://www.eurosurveillance.org/ ViewArticle.aspx?Articleld $=20419$

Article published on 07 March 2013

On Monday 4 March 2013, the European Centre for Disease Prevention and Control (ECDC), launched a new interactive database and published its first report on surveillance of antimicrobial consumption in Europe.

ECDC collects data on antimicrobial consumption from 29 European Union (EU) and European Economic Area (EEA) countries through the European Surveillance of Antimicrobial Consumption Network (ESAC-Net), which is a Europe-wide network of national surveillance systems. The report presents data for 2010 from the community (primary care) and the hospital sector.

The interactive database provides public access to tables, maps and figures on antimicrobial consumption down to the 4 th level of the anatomical therapeutic chemical (ATC) classification. Additionally the interactive database contains antimicrobial consumption data from 1997 to 2009. These data were collected by the EU-funded European Surveillance of Antimicrobial Consumption (ESAC) project before it was transferred to ECDC in July 2011 and was renamed ESAC-Net. ECDC will shortly update the interactive database with data for 2011 and 2012.

The report and the interactive database include data for three major groups of antimicrobials: antibacterials for systemic use (ATC group J01); antimycotics for systemic use and antifungals for systemic use (ATC groups J02 \& Do1BA) and antivirals for systemic use (ATC group J05).
The report shows that, on average, $90 \%$ of antibacterials for systemic use are consumed in the community i.e. outside hospitals. In 2010, the consumption of antibacterials for systemic use varied by a factor of 3.5 between the participating countries: from 39.4 to 11.1 Defined Daily Doses (DDD) per 1,000 inhabitants and per day. The increasing trend in consumption of antibacterials for systemic use observed in the community in previous years was discontinued in 2010. For the first time, data on antimycotics and antifungals for systemic use from the hospital sector are presented.

In order to make valid inferences regarding the determining factors behind the observed changes and trends in antimicrobial consumption, additional data would be needed on prescriptions, indications for prescribing as well as information on national programmes on the prudent use of antimicrobials.

ECDC aims to provide timely data and independent reference information on antimicrobial consumption in Europe, to support EU/EEA countries in their efforts to promote rational use of antimicrobials, and to prevent and control antimicrobial resistance.

The first ESAC-Net report is available for downloading on the ECDC website: http://ecdc.europa.eu/en/ publications/Publications/antimicrobial-antibiotic-consumption-ESAC-report-2010-data.pdf

The ESAC-Net interactive database is accessible on the ECDC website: http://ecdc.europa.eu/en/activities/surveillance/ESAC-Net/database/Pages/database.aspx 